\title{
Erratum: Tracking genomic hydroxymethylation by the base
}

Gilles Salbert \& Michael Weber

Nat. Methods 9, 45-46 (2012); published online 28 December 2011; corrected after print 18 February 2013

In the version of this article initially published, ref. 1 contained an incorrect volume number and year. The errors have been corrected in the HTML and PDF versions of the article.

\section{Corrigendum: Annotated high-throughput microscopy image sets for validation}

Vebjorn Ljosa, Katherine L Sokolnicki \& Anne E Carpenter

Nat. Methods 9, 637 (2012); published online 28 June 2012; corrected after print 25 February 2013

In the version of this article initially published, funding information was not included. The work was funded by US National Institutes of Health grant R01 GM089652. The error has been corrected in the HTML and PDF versions of the article.

\section{Erratum: Single-chromosome transcriptional profiling reveals chromosomal gene expression regulation}

\author{
Marshall J Levesque \& Arjun Raj \\ Nat. Methods 10, 246-248 (2013); published online 17 February 2013; corrected after print 27 March 2013 \\ In the version of this article initially published online, several bars in the bottom bar graph in Figure $2 \mathrm{c}$ are missing their fill color. The error \\ has been corrected in the PDF and HTML versions of this article; this error did not occur in the print version.

\section{Erratum: Critical assessment of automated flow cytometry data analysis techniques}

Nima Aghaeepour, Greg Finak, The FlowCAP Consortium, The DREAM Consortium, Holger Hoos, Tim R Mosmann, Ryan Brinkman, Raphael Gottardo \& Richard H Scheuermann

Nat. Methods 10, 228-238 (2013); published online 10 February 2013; corrected after print 1 April 2013

In the version of this article initially published, the affiliation listed for Marc Strickert was incorrect in the PDF. In the HTML, a numbering error caused a mismatch of affiliations to authors for members of the FlowCAP and DREAM consortia. The errors have been corrected in the HTML and PDF versions of the article.

\section{Corrigendum: Critical assessment of automated flow cytometry data analysis techniques}

Nima Aghaeepour, Greg Finak, The FlowCAP Consortium, The DREAM Consortium, Holger Hoos, Tim R Mosmann, Ryan Brinkman, Raphael Gottardo \& Richard H Scheuermann

Nat. Methods 10, 228-238 (2013); published online 10 February 2013; corrected after print 1 April 2013

In the version of this article initially published, the affiliations or names of of the following authors were not correctly communicated to the journal: Nicolas Guex, Heikki Huttunen, Tobias R. Kollman, Tapio Manninen, Matti Nykter, Pekka Ruusuvuori and Michael Seifert. The errors have been corrected in the HTML and PDF versions of the article. 